\title{
Arterial spin-labeled MRI for diagnosis and monitoring of AD
}
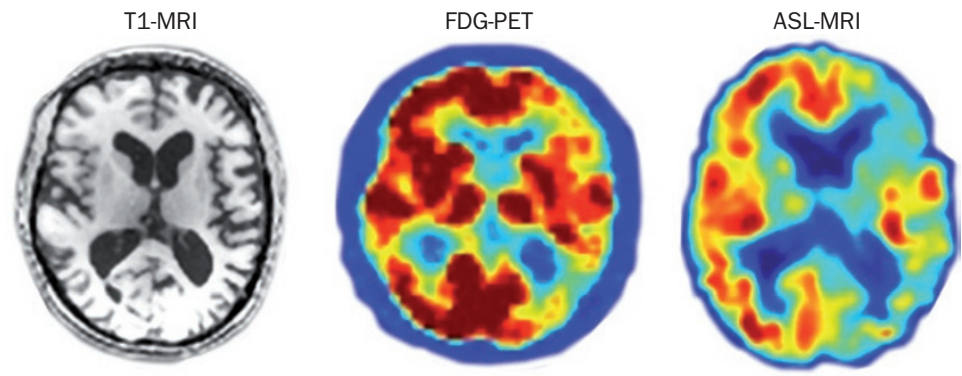

Images of the brain of a patient with Alzheimer disease showing anatomy (T1-MRI), glucose metabolism (FDG-PET), and cerebral blood flow (ASL-MRI). High metabolism or blood flow are indicated by red colors and low metabolism or blood flow by blue colors. Images courtesy of J. Detre.

Arterial spin-labeled perfusion MRI (ASL-MRI) has comparable sensitivity and specificity to fluorodeoxyglucose PET (FDG-PET) imaging to detect functional changes in the brain and provide a basis for the diagnosis of Alzheimer disease (AD), according to two recent studies. The use of ASL-MRI could reduce both the cost of imaging and the time to diagnosis in patients with $\mathrm{AD}$, and may enable faster screening of patients who present with cognitive deficits.

Individuals with $\mathrm{AD}$ show specific patterns of altered resting-state glucose metabolism, which is routinely imaged using FDG-PET. ASL-MRI measures cerebral blood flow, which is coupled to neural activity. "We hypothesized that the patterns of reduced cerebral blood flow in patients with AD measured by ASL-MRI would match the patterns of reduced glucose metabolism measured by FDG-PET," explains John Detre from the University of Pennsylvania, PA, USA, who was the senior author on both papers. The use of ASL-MRI-measured cerebral blood flow as a biomarker in $\mathrm{AD}$ is appealing, as the technique is noninvasive, and MRI is cheaper and more widely available than FDG-PET.

In the first study, Musiek et al. used a novel protocol to obtain concurrent ASL-MRI and FDG-PET images in 17 patients with AD and 19 healthy controls. "We measured glucose metabolism and blood flow at the same time by injecting the FDG tracer into patients during the ASL-MRI scan," explains Detre. On visual inspection of the images, ASL-MRI enabled the clinicians to identify the patients with AD with an identical diagnostic sensitivity and specificity to FDG-PET imaging.

Reporting in Neurology, Chen et al. used a voxel-based analysis of the same patient images to confirm that the patterns of regional cerebral blood flow and glucose metabolism had a statistically significant overlap. When compared with images from healthy controls, ASL-MRI enabled detection of functional deficits in specific regions of interest in the brains of patients with $\mathrm{AD}$, again with a similar sensitivity to FDG-PET imaging.

"These findings confirm prior work that suggested ASL-MRI could detect brain function changes in $\mathrm{AD}$, and are the first to directly compare ASL-MRI and FDG-PET in the same cohort," says Detre. "ASL-MRI can be obtained in conjunction with any routine brain MRI, which could be useful in screening for $\mathrm{AD}$ in patients who present with cognitive complaints, or potentially in screening for any brain disorder."

Katy Malpass 